\title{
Hacia una Filosofía de la Educación.
}

\section{INTRODUCCION}

\section{1.-LO QUE ES LA FILOSOFIA.}

Sabido es que en su acepción etimológica, la Filosofía significa literalmente "amor a la sabiduría", o "aspiración a la sabiduría ". Así lo concibieron primitivamente los griegos antiguos, allá en el despertar de su conciencia reflexiva. Muy pronto, empero, ese sentido original desaparece, para abarcar al mismo tiempo que ese "amor" (tendencia hacia el saber teórico), la "posesion" efectiva de la sabiduría misma, lograda como resultado de los esfuerzos y tanteos del hombre para alcanzarla. Esta etapa inicial y básica de la evolución de la idea de la Filosofía se cumple en el lapso comprendido entre la aparición del primer pensador jónico Tales de Mileto, hasta el gran humanista Sócrates.

Desde entonces, se puede decir, la Filosofía muda a cada rato de ropaje, no obstante conservar su indumentaria funđamental ("amor" y "posesión" de la sabiduría), paralelamente al desarrollo del pensamiento en el decurso del tiempo, hasta el punto de que, a la fecha, _andados más de 
veinte siglos de historia - podemos recoger de aquí y allá, de boca de los mismos "filósofos", multitud de definiciones, fruto del modo personal cómo cada uno de ellos se octipa en filosofar. De esta suerte parece existir una aparente confusión de conceptos alređedor de la Filosofía, ya que en cada "filósofo" y en cada "sistema filosófico" oteamos una noción distinta de ella, dado en gran parte por la dirección en que se ejercita la actividad filosófica o la clase de objetos hacia los que vira la Filosofía en cada pensador.

Un notable filósofo, Guillermo Dilthey, que procuró deslindar la esencia de la Filosofía, en un ensayo acucioso (I), sirviéndose de la inducción histórica, concreta su opinión acerca de lo que acabamos de decir, en estos términos: "El nombre filosofía o filosófico tiene, según la época y el lugar, significados distintos, y las creaciones espirituales que han recibido este nombre por sus autores son tan diversas, que parecería que las distintas épocas hubieran asociado a la hermosa palabra filosofía, acuñada por los griegos, imágenes espirituales ]siempreadistintase Pues unos entienden por filosofía la fundamentación de las ciencias particulares; otros amplían este concepto añadiendo a la fundamentación la tarea de deducir de aquélla el conjunto de las ciencias particulares; o la filosofía se circunscribe al complejo de estas ciencias; otras veces se la define como la ciencia del espíritu, la ciencia de la experiencia interior; finalmente se entiende también por ella la comprensión de la conducta de la vida o la ciencia de los valores universalmente válidos". . Después agrega el mismo Dilthey que la Filosofía es una autorreflexión del espíritu y a la vez una teoría del saber. Siguiendo este mismo pensamiento de Dilthey, otro inves-

(1).-G. Dilthey.-- "La esencia de la Filosofia",-Ed. Losada,-Buenos Aires.- 
tigador, J. Hesen, (2) define la Filosofía diciendo que es "una autorreflexión del espíritu sobre su conducta valorativa teórica y práctica, y a la vez una aspiración al conocimiento de las últimas conexiones entre las cosas, a una concepción racional del universo". Y recientemente, un pensador notable, Aloys Müller, (.3) alude también a las discrepancias en torno a esta cuestión, y reúne, como muestra, muchas y contradictorias definiciones de la Filosofía; concluve afirmando al término del tratamiento de los temas propiamente filosóficos (Ontología, Lógica, Teoría del Conocimiento, Etica, Estética, Religión, Metafísica y Concepción del Universo) que la Filosofía es una ciencia que se ocupa del estudio reflexivo de los valores y de lo suprasensible.

Dejando aparte todo lo anterior, y con propósito de llegar por nuestra nropia cuenta a to que hace que la Filosofía sea cabalmente Filosofía, y no otra cosa,-por ejemplo, Física o Astronomía - hemos de fijarnos en su núcleo, que resplandece más allá de cada filósofo y đe cada sistema filosófico. Por de pronto curge tener en cuenta que el hombre hace Filosofía en procura de un saber objetivo, universal y sistemado, que valga como un saber último, definitivo y esencial acerca del hombre, del mundo y en general de la vida. Para esto es que filosofamos, dice Aníbal Sánchez Reulet (4). Por eso filosofar, en el fondo, equivale a "teorizar". Esta palabra posee, etimológicamente, un significado muy rico. "Teorizar" es al par que un pensar con ánimo de saber lo que las cosas son, un esfuerzo mental para explicarnos porqué y cómo estas cosas son. De ahí que, al teorizar, lo que hacemos es buscar un conocimiento que nos permita hallar una explicación racional y objetiva (en sí y pa-

\footnotetext{
(2).-J. Hesen.-Teoría del Conocimiento.-Ed. Losada.-Buenos Aires.

(3).-A. Miiller.-Introdueción a la Filosofí.-Ed. Calpe.-Buenos Aires.

(4).-Aníbal Sáchez Reulet.- "Raíz y destino de la Filosofia", Tucumán.
} 
ra sí) de cuánto es y acontece ante nosotros. Teorizar viene de "teorein", que en el idioma de Platón es algo así como observar, ver o contemplar mentalmente las cosas. Todo aquél que teoriza, o que quiere teorizar, tiene que adoptar una postura de observación, de visualización o contemplación mental. Sólo así es posible un auténtico filosofar. Y sólo de ese modo, también, podemos columbrar un concepto de la Filosofía, y de la actitud filosófica, en cuanto entrañan una actividad teorética o teorizadora.

No es ello tođo, sin embargo. La disposición filosófica, o lo que es igual, la actitud teorética, con miras de hacer FiInsofía, obliga a un colocarse fiente a frente a aquéllo que debe constituírse en materia de la contemplación mental $o$ reflexiva. Es run situarse en relación de polaridad con respecto al foco de incidencia de la actitud teorética; mejor dichio, una correlación funcional polarizada entre el suieto teorizante y el objeto teorizado. Y esto apareja, o tiene que aparejar forzosamente un empuje de extrañamiento, es decir un hacerse extraño u otro por parte del sujeto que teoriza, a fin de distinguir claramente ef objeto teorizado, o sea el objeto que se "enfrenta" y que es "visto" en la reflexión teorética como una realidad extraña y diferente. Esto no sería factible si el sujeto teorizante no experimentara previamente una afección recóndita de asombro, sorpresa o ađmiración como camino necesario hacia la extrañeza que debe ponerle en actitud teorética.

Platón y Aristóteles fueron quienes vieron en la admiración el comienzo de la Filosofía. "La turbación es un sentimiento propio del filósofo", decía Platón (5), y Aristóteles a su vez añade que "debido a su admiración comenzaron y comienzan los hombres a filosofar.... El hombre se sien-

(5).-Platón.-Teetetes. 
te perplejo y maravillado, considérase ignorante.... por eso filosofaron con el fin de salir de la ignorancia"... (6). No se crea sin embargo que cualquiera admiración es fuente de Filosofía. Lo es tan sólo aquélla que al mismo tiempo que encierra la sorpresa del alma infantil ante lo abigarrado y abundoso de las cosas, supone un quedarse en suspenso ante las propias preguntas que surgen como consecuencia del asombro; emoción que, por otra parte, transparenta un franco sentido de los problemas planteados por el ser y devenir multitudinarios del mundo y de la vida, dentro de cuyo engranaje ingente se contempla el hombre mismo. De ahí el acento problemático que ostenta la admiración verdaderamente filosófica. Quien problematiza las cosas y en general todo cuánto se le preesenta, en busca de un conocimiento verdađero, está filosofando. La problematicidad filosófica hunde sus raíces en la duda, especialmente en la duda me. tódica, como diría Descartes, o sea en la conciencia, mas o menos clara, de las dificultades en encontrar una respuesta adecuada o satisfactoria a las preguntas brotadas de la admiración. La Filosofía, por eso, proviene de una confrontación y tratamiento de problemas diversosoplanteados por otras tantas interrogaciones hijas de una turbación elevada y legítima ante el misterio del Cosmos. De ahí que ser filósofo es percibir problemas donde otros no ven si no hechos naturales que octrren por que sí, al azar acaso, y que son, efectivamente, tal cual se muestran o parecen ser; y es además sentir imperativamente el afán de encararse a las cosas o fenómenos, para quitárselas la máscara que cubre su faz auténtica y desnuda. Para el hombre corriente o vulgar, las cosas pasan casi
desapercibidas. Estas se halla como en penumbra ante sus

(6).-Aristóteles,-Metafísica, 
ojos. De vez en cuando una leve ráfaga de claridad o de asombro surge en torno; es entonces que mira un poco mejor y se percata de pequeños detalles, pero en su mayoría triviales y de poca monta. Por eso su estado natural es creer que las cosas están allí, ante él, tal cual son, por lo que su conocimiento es supuestamente "real" y por ende exacto. Algo más: el hombre vulgar mismo está como "perdido" o "abandonado" entre las cosas que le circundan, como si fuera una cosa más; y él existe así, en la existencia, como algo que se ignora y que por ignorarse precisamente vive como fuera de sí, como si fuera una presencia extraña a sí mismo. Esto le basta ciertamente, porque, en efecto, no necesita más para vivir y dejarse vivir; por lo cual vive sin inquietarse mayormente y sin querer hacer nada para que su vida sea otra cosa de lo que es. Por manera que, como diría Martín Heidegger, (7) se puede decir de este hombre que es un objeto más entre los tantos que existen, una cosa más encajada dentro de la pluralidad de las cosas que están en el mundo, lejos de sí. Es esta la etapa ingenua y primitiva en la vida del individuo y en la historia del hombre en general.

No sucede lo mismo ya en el hombre de ciencia. Este experimenta una manifiesta curiosidad por éstas o aquéllas cosas. Para él, el mundo no ostenta la firmeza que tiene para el hombre vulgar o corriente. Al contrario, atisba que las cosas vacilan entre ser esto o lo otro, y que su aparecer y desaparecer tiene lugar según ciertas formas constantes, mas o menos uniformes. Pretende ir entonces un poco más allá de la cáscara que encubre los fenómenos, para descubrir su causa u origen, asi como las leyes a que obedecen en șu ser y transcurrir. En esta investigación avanza

(7).-Alberto Wagner de Reyna,-La ontología fundamental de Heidegger-Lima. 
con cautela, paso a paso, cuidándose de no ser engañado y deteniéndose ante tal o cual grupo de hechos; de modo que el saber que adquiere por esta vía, es siempre un saber circunscrito a determinada área de la realidad, que solaniente cala cierta profundidad y que fragmenta la unidad primordial de todo lo que es en animales, plantas, minerales, astros, hombres, movimientos físicos, etc. todo ello lo mejor ordenado y dispuesto en apartados diferentes, bajo membretes tales como Zoología, Botánica, Física, Química, Mineralogía, Antropología, Psicología y demás ciencias particulares. De este modo el hombre de ciencia se queda con puñados de conocimientos parcialmente unificados, catalogados con el máximo rigor posible y subordinados en su mayoría, no en su totalidad, a leyes de carácter causal, de mera succsión o de coexistencia, según los casos.

En el que hace Filosofía, o en el que filosofa, se ensancha la visión del hombre de ciencia. El filósofo es un tipo especial de hombre de ciencia, que se remonta más allá de la mera causalidad y busca el núcleo unitario del ser. El filósofo no se turba antes éstas o aquéllas cosas simplemente, sino ante la totalidad de fos fenómenos, ante su sentido unive1:sal e intemporar.Ea extraneza del filosofo resulta así abso.luta e integralista. $Y$ esto porque al caminar por entre la problematicidad de la totalidad existente, persigue la raíz espermática y radical de ésta. De ahí su incansable interrogar al mundio y a sí mismo. Es como un niño grande, pero ya maduro y con la conciencia en eterna vigilia, que tiene la manía de buscar el interior de las cosas, de sorprender realidades ocultas a la mirada superficial, yendo de aquí para allá permanentemente curioso e insatisfecho de todo, hasta de sí propio. Preguntas como éstas danzas en su cerebro: "¿Qué es lo que hay detrás de las cosas?" “¿De dónde venimos?” 
“¿Adónđe vamos?” “¿Qué valor tiene la vida, nuestra vida, la vida de cada uno?" "¿Cómo y por qué necesitamos justificar nuestra conducta?" "¿Qué debemos hacer?" "¿Qué es el ser?" “¿Qué son los valores en sí?” "¿Existe un Ser Supremo como causa y sostén del Universo?” “¿Hay una conexión íntima y funcional del hombre con la esencia del mundo?” “¿Qué es lo espiritual?”.

Así es cómo el filósofo camina sobre el terreno de la Filosofía, estremecido y descontento, teniendo que hacer frente a cada paso a las preguntas que le salen al encuentro desde los limbos de lo desconocido. Ya no se contenta con el saber empírico, contingente y caótico del hombre vulgar o corriente; ni tampoco con el saber sistematizado y fundamentado, pero parcializado, incompleto y superficial del hombre de ciencia, que en lugar de unir destruye más bien la conexión simbiótica de las cosas. A lo que el filósofo aspira es a desvelar la "razón suficiente" de lo que es y acaece, o sea el porqué y la significación iltima que subyace debajo del cambio y la movilidad perpettas. Por eso tiene que abandonar continuamente las suposiciones, las explicaciones provisionales y los prejuicios, si qûere arribar con éxito a lo esencial, a lo que permaneee yy flòta tmás allá cle da vibración epidérmica y efímera. Tal el motivo por que el saber filosófico, al fin y al cabo, tenga el carácter de un saber sin supuestos y además de un saber esencial.

El hombre ha entrevisto, desđe Sócrates, que la rendija por donde puede infiltrarse en el Ser, es él mismo. El hombre es, por antonomasia, el camino, la verdad y la vida. Por eso el conocimiento del hombre nos conduce al conocimiento de la estructura esencial del mundo. El mandato socrático vuelve a recobrar así la plenitud de su vigor y de su actualidad. El hombre es el punto céntrico de la Filosofía, su 
clave, el eje en torno al cual despuntan todos los problemas que confronta aquélla. Conciente de esto, el filósofo se es.mera, o debe esmerarse en preparar. y afinar sus instrumentos cognitivos, para lo que debe mantenerse en el primer plano de la objetividad, precavido y alerta para no permitir que le desvíen de su ruta, ni menos enturbien su visión serena y penetrante, los espejismos de la prestuposición antojadiza.

Sólo así es posible la Filosofía y es posible también el auténtico filosofar. Toda meditación filosófica tha de encuadrarse dentro del cauce de una actitud teorética afanosa de realidad, mejor aún, debe ser una caza de esencias. De ahí que la Filosofía puede ser definida en último término como una ciencia teorética de esencias. Y tanto aquélla como el filósofo que la hace, han de estar exentos de los supuestos que desnaturalizan la contemplación reflexiva, y han de estar también empujados por una determinación de ir siempre más állá, hacia una objetividad cada vez mayor, más plena, más universal y necesariamente válida. Lo cual no significa que el filósofo haya de ignorarse a sí mismo como hombre. Al contrario, él está en todo momento presente al modo de flecha indicadiora de la ruta a seguirse. Ya dijimos que la Filosofía surge del hombre yc se extiende alrededor del hombre; y hacia el hombre también debe volver para verificar sus resultados. $\mathrm{Y}$ es que los problemas del hombre consuenan con los del mundo y se equivalen ontológicamente, por lo solidarios que son y por constituir tanto aquél como éste un sólo gran problema para la Filosofía. De este modo la Filosofía, y en general todo filosofar se mueve en función siempre de lo humano, por lo que su contenido deviene en edificio sólido que encierra el secreto suprasensible del hombre y del mundo.

Para concluír, pues, y resumiendo lo que precede, hemos de decir que Filosofía es no sólo la actitud teorético-re- 
flexiva encaminada hacia la captura de esencias, si no también su resultado, su producto, concretado bajo la forma de un saber esencial; pero —entiéndase bien- sólo en tanto este producto brota legítimamente de aquélla actitud como tal. El saber que no es resultado necesario de una actitud enrumbada hacia la aprehensión đe esencias, no es propiamente saber filosófico, y por ende no puede ser llamado Filosofía. Hablamos de Filosofía, en la acepción que damos arıú a esta palabra, no sólo cuando existe una búsqueda teorética de esencias, si no cuando ha habido aprehensión de éstas en un saber sistemado, objetivo, último, necesario y universalmente valedero; saber que, además, puede servir de base y fundamento a todas las demás ciencias đe hechos. Por eso es comprensible la Filosofía como una ciencia teorética de esencias. Y en esto se diferencia la Filosofía de las otras Ciencias, que son Ciencias fácticas o de hechos.

\section{2.-IA FIJ,OSOFIA COIMO NECESIDAD VITAL.}

De las consideragiones que antecèden, cabe presumir el rol de la Filosofía en la vida humana. El hombre hace Filosofía, como otras tantas cosas - arte, religión, derecho, literatura, etc., precisamente porque está en su propensión óntica cl hacer todo eso. Y está en su ser el hacer Filosofía, por que necesita y está vitalmente interesado en ello. Es un interés y una necesidad insobornable e insoslayable. El hombre, en efecto, en el decurso de su existir, se ve en la urgencia de interrogar, al anđar de sorpresa en sorpresa. En un principio tal vez sus preguntas inciden sobre asuntos triviales e insignificantes. Mas muy luego, cuestiones más espinosas le requieren por todas partes. De buena o mala gana, quiéralo o) nó el hombre, durante el proceso de su paulatina maduración interior, recurre inevitablemente las etapas del hombre 
ingenuo o vulgar, del hombre de ciencia y del filósofo. No hay quién se quede en el primer estadio, entregado a la espontaneidad y a la aceptación confiada de la realidad de sus percepciones. Pronto entra cada cual en el período del hombre de ciencia, que desconfía de sus sentidos y busca el nexo causal entre unos y otros fenómenos. Y al cabo, más tarde o más temprano, cae en la edad del filósofo, o cuando menos del filosofador; edad tardía, es cierto, pero edad hacia la cual llega el hombre psicológica e históricamente. Y esto porque tiene que interrogar forzosamente en el curso de su marcha a través del tiempo y del espacio, y debe hacerlo por que está en su destino el verse ante problemas que le apremian y que por hallarse generalmente ensartados los unos en los otros formando una cadena que va de lo inmediato y contingente a lo remoto y estable, exigen una resolución integradora, aún cuando, en un principio, sea indispensable abordarlos uno a uno, comenzanđo por los más pequeños que se hallan, por así decirlo, a la mano, para ascender 1uego hasta lo\$imás graves detrascendentes que comportan preguntas más complejas y dilatadas y que, desbordando el círculo de nuestras exneriencias individuales y sensibles. rozan los linderos supraindividuales e inexperimentables de la realidad metafísica.

Lo efectivo es pues que el hombre se ve alguna vez en el trance de tener que filosofar. Y filosofa, porque interroga, teoriza, problematiza. Pregunta, en razón de precisar un conocimiento acerca de lo que en esencia son las cosas y en general el Universo junto con el cual convive y coexiste en simbiosis permanente. Problematiza, o sea convierte en problemas las situaciones propias y del mundo, los procesos subjetivọ y objetivos, en vista de no ser dueño de un saber esencial que le permita salir de las dudas e incertidumbres 
y le ofrezca una base segura para su acción intencionađa y prospectiva. $Y$ teoriza, por último, porque se esfuerza en contemplar en su esencia o "eidos" el medio circundante y aún la totalidad cósmica, tanto para determinarse adecuadamente en su conducta, como para penetrar en el valor y sentido de cuánto es y existe y poder hallarse así en condiciones de justificar ante sí y los demás su pensar, su sentir, su querer, su valorar y de justificar, si es posible el ser y acontecer universales.

De esta manera al hacer Filosofía, el hombre intenta iluminar la entraña problemática de su existencia. En parte esto por aue el hombre no Duede mantener su categoría liumana sin un motivo que le asista y responda de lo aue hace $\mathrm{v}$ de lo aue es. La motización consciente es reauisito capital de su sunervivencia ontologica rlentro de la esencia hitmana y debe hallarse a la hase de sus decisiones más auténticas y aún de su inactividad o pasividad. si por acaso en ello reposa. Por lo cual alouna razón suficiente han de tener siempre su hacer en no hacer vitales. De ahí la irrefrenab!e orientación del hombre hacia la Filosnfía. Esta tendencia y necesidad de la "Filesofía se torna másepatente si consideramos que el homhre no se esmera solamente en perseverar en su ser, como diría Spinoza (8). si no aue su propósito es siempre ser más de lo que es, realizarse de un modo cada vez más acabado y completo, desenvolver enteramente sus ricas posibilidades evolutivas, superando su actual e inconcluso ser. Y ello es posible únicamente en cuanto filosofa, vale decir en tanto cala más y más hondo en la raigambre esencial de su organización y de su devenir, y pone de relieve su excelsa posición espiritual en el Cosmos. (9).

(8).-D. Spinoza. Etica.

(9).-M, Scheler.-El puesto del hombre en el Cosmos. 
Es indudable pues que en la medida en que el hombre hace Filosofía, sortea mejor las dificultades y cuestiones que a lo largo de su vivir encuentra, y sobre todo salva la gran dificultad y la gran cuestión de su propia existencia personal. Lo primero, en efecto, con que tropieza el hombre es con el obstáculo y el problema de tener que subsistir a torla costa, según tales o cuáles módulos y en la mejor dirección que sea capaz de intuír, a fin de arribar con éxito a la capa de mayor rango ontológico. $\mathrm{Y}$ para esto le es forzosamente indispensable filosofar en más alto grado siempre, aunque las más de las veces lo haga con prosaísmo o poca elevación, ya que se halla presionado por lo que Maeterlink califica de "trágico cotidiano". X así el hombre ha menester en todo instante de la Filosofía, sea para enderezar con más propiedad su actividad teorética, sea para vindicar su funcion valorativa o para humanizar su comportamiento práctico y prospectivo; de este modo podrá alcanzar el plano de litz de la mayor conciencia y moverse en un ambiente acorde con los ideales y las normas que infunden sobresaliente valor y significación a la historia del hombre sobre la tierra. Tal la razón y la finalidad del quehacer filosófico. La Filosofía es por ello una necesidad vital imperiosa e ineludible.

\section{3.-VIVENCIA DE LA FILOSOTIA}

La noción de vivencia arranca del pensador alemán Guillermo Dilthey ( IO), quién la empleó para designar el "lazo de vida" o de conexión metafísica del filósofo con las cosas, en virtud de una relación esencial con la totalidad del Universo estimado y experimentado singularmente como realidad espiritual omniabarcante y divina. Muy luego, sin em-

(10).-G. Dilthey.-Introduceión a las Ciencias del Espíritu. 
bargo, en aquel mismo pensador, esa noción es extendida hasta comprender en su referencia a toda forma de comunión íntima e irracional del hombre con el mundo y de modo particular con la vida una e infinita, una de cuyas múltiples reverberaciones es él mismo.

El concepto de vivencia se expande posteriormente, y filósofos que vienen después de Dilthey, como Scheler (II), Spranger ( I2) y Husserl (I3), principalmente, lo dotan de una resonancia más concreta y si se quiere especial. Así en Scheler llámase vivencia al hecho de vivir irracionalmente contenidos objetivos de la más diversa índole; en Spranger es algo así como una conmoción profunda que sacude los cimientos de la vida interior y se prolonga, al modo de una huella de vibración imborrable, bajo la forma de una energía vital potenciada que debe servir para la edificación del espíritu subjetivo, fomentando la evolución y estructuración personal de los valores; $y$ en Husserl la vivencia es todo lo que se encuentra en el flujo de lo vivido y experimentado y dentro de la cual se insertan los elementos y aprehensiones psíquicas, siendo, por tanto, base pura de estos; la vivencia viene a ser así lo tenido primordialmente en fa corriente pura del vivir subjetivo.

Para captar mejor el sentido de la palabra vivencia, dentro de la Filosofía y Psicología actuales, hemos de indicar su origen etimológico. Con el término vivoncia el filósofo español José Ortega y Gasset quiso traducir al castellano la palabra alemana "Erlebnis". Este vocablo alude más que al simple y mero vivir en sentido general - lo que en alemán se designa con la palabra "Leben"-, o sea al ingenuo dejar vivir

(11).-M. Scheler--Esencia y formas de la simpatía.

(12).-E. Spranger.-Psicología de la edad juvenil.

(13).-E. Hussert.-Investigaeiones Lógicas. 
la vida en nosotros; significa, sobre todo, vivir algo, es decir el vivir de lo vivido mismo, la vida vivida en función intencional de aquéllo que es vivido y hacia lo que cabalmente apunta el vivir como fenómeno anímico. De este mođo en la vivencia poseemos subjetivamente un contenico intencional que llena, por así decirlo, nuestro vivir. Por lo cual, pues, la vivencia, como acto puro de dirección psico-espiritual implica la vida que es vivida por nosotros en correlación funcional (intencional) de aquéllo que es vivido como materia u objeto de la vivencia misma, y que por eso aparece identificada con ésta. No supone ello que haya conciencia, o sea un copocimiento de lo vivido en la vivencia. Al contrario, la vivencia puede transcurrir y transcurre, en efecto, comunmente, al margen de la conciencia. Esto por que la conciencia es un fenómeno de segundo grado é involucra un "saber" de la vivencia, pero nunca la vivencia estrictamente como tal. Es que la vivencia es siempre un proceso íntimo puro, ingenuo, primitivo, cual un oleaje recóndito que emerge en la dinámica de nuestro ser, al contacto pático - nó gnóstico- con la pulsación interior del mundoy de la vidadinfinita.

La vivencia es, por tanto, un proceso subjetivo complejo que se actualiza más bien allá en los estratos afectivos de nuestra existencia psico-biológica. De ahí el dejo irracional y primigenio de la vivencia. Es ella quién nos pone verdaderamente en comunicación con las realidades más sustantivas y últimas. Nosotros podemos pensar cualquier cosa o hecho, podemos imaginárnosla, representárnosla mentalmente; empero mientras no la tengamos realmente en la vivencia, mejor dicho, en tanto no la poseamos vivencialmente, como una presencia fresca e impalpable a la que hospedamos mágicamente, permanecerá en principio desconocida y distante para nosotros. Por eso la vivencia es la intercesora auténtica y 
autorizada entre el mundo exterior y nuestro mundo interior; y así entonces la vivencia resulta el medio directo e inmediato de comunión de lo hondo con lo hondo, el puente que nos conecta con la pulsación metafísica de la vida universal, viviéndola en nosotros en fusión mística, conviviendo con su vaivén inmarcesible e ilimitađo.

Consecuentemente con lo que antecede, estamos en condiciones de referirnos a la vivencia de la Filosofía. En efecto, sólo en tanto tengamos una vivencia de la Filosofía misma, podemos entrar en contacto con ella y saber, por experiencia personal, de su realidad, de su valor y de su significación vital y espiritual. Podemos tener, con mayor o menor vaguedad, alguna idea de la Filosofía; - y de hecho cada cual la posee - pero nunca llegaremos en verdad a sus dominios si no la tenemos posítivamente en la vivencia, vale clecir, si no la vivimos en nuestro interior, si no la convertimos en carne propia, en nervio, en sangre; si no convivimos con sus problemas, con sus esfuerzos, con sus tanteos en lo ignoto, viajando por su inmenso territorio y contemplando por nosotros mismos sus montañas, sus valles, sus llanuras, sus picos elevados, sus climas, sus abismos, etc., como bien recalca Manuel Garcia Gorente (14).

Lo importante, pues, - como dice este notable maestro español- no es que nosotros recojamos de aquí o allá determinadas fórmulas o pensamientos filosóficos, pensados por algún filósofo o que están en algún sistema filosófico. Seguramente la frase gramatical o verbal tendrá algún sentido para nosotros. Pero, en el fondo y a pesar del apoyo que nos preste la memoria, estaremos lejos de la realidad a que se refieren aquéllas fórmulas o pensamientos, puesto que éstos

(14).-M. Gareía Morente--Leceiones preliminares de Filosofía. 
diríase, son representaciones alegóricas de algo muy hondo y vivo, o cuando menos moldes intelectuales en que se quiso o se intentó vaciar y a la vez fijar el flujo incoercible de la vivencia filosófica correspondiente, no conteniendo, por ello, la vivencia misma que los dió origen. Por lo cual se hace necesario, si pretendemos llegar a la Filosofía, que cada uno de nosotros, por su propia cuenta y riesgo, se ponga a filosofar, a vivir y a estar en trato con las cuestiones filosóficas. Si no hacemos así, caemos en el error craso de querer aprender a nadar, sin echarnos al agua. Ciertamente, como dice Hegel, siempre es útil en algo adquirir algunas nociones previas de la Filosofía para podernos lanzar con éxito, posteriormente, a filosofar por nosotros mismos. Aún así, sin embargo, jamás se podrá afirmar con rotundidad que hacemos Filosofía por el hecho de repetir mecánicamente determinadas fórmulas o concepciones filosóficas, como tampoco podemos garantizar nuestra aptitud para la natación con sólo habernos enterado, en un manual, de los movimientos requeridos, sin habernos metido en ningún momento al agua, poronuestra propia euenta, para nadar.

Es precisopenetrar efectivamente en el reino de la Filosofía, es filosofando, ante todo y sobre todo. Así pronto podremos saber, por personal experiencia, que la Filosofía es la tarea más elevada a que podemos entregarnos y la que tiene también más hondas raíces vitales y espirituales. Así vivida y creada, sentida y padecida, la Filosofía ya no será un cuerpo cerrado y extraño por donde circulan jeroglíficos, frases abracadabrantes o esquemas intelectuales yertos, sino que se nos presentará como la encarnación de nuestras propias y más altas preocupaciones y por ende como nosotros mismos en 
cuánto aquélla refleja nuestro pensar, nuestro sentir, nuestro querer, nuestro valorar, etc.

\section{4.-IA FIJOSOFIA DE LA EDUCACTON.}

Concordante con todo lo expuesto, ¿qué debe ser entonces y qué es lo que persegtuirá una Filosofía de la Educación? Si tenemos en cuenta lo que dejamos dicho acerca de lo que es la Filosofía en sí, fácil es percatarnos de los propósitos de una Filosofía de la Educación. En este caso, la educación constituye el foco y la meta del quehacer filosófico. La educación no sólo es un hecho social y un hecho histórico en la evolución cultural del hombre, sino que, además y sobre todo, es un ideal de superación y perfección. Por eso la educación es algo que hacemos; pero, al mismo tiempo, es también una dirección de este hacer. El hacer eđucativo llévase a cabo dentro y en función de la vida y del mundo, o mejor aún, sobre un ámbito específicamente delimitado del espacio y del tiemno. Es este ámbito lo que constituye propiamente el "mundo" p lac"vicla" humana, vles sobre el mismo ámbito que se ciemnen las directivasnideales, y normativas que señalan el derrotero de su hacer educativo. Empero no es ello todo. sino que hemos de tener en consideración asimismo las líneas universales, las grandes constantes en que se mueve el ritmo subjetivo de la vida humana; y sólo en tanto procedemos así podremos trazar el círculo de la educación como hecho y como ideal, en correlación dialéctica con los elementos externos.

Confrontaremos entonces, en primer lugar, y en sus lineamientos generales, la estructura biológica del hombre en función tanto del escenario geográfico como de la particular atmósfera histórico-social en que se mueve y con los cuales está conectado dialécticamente en el curso de sus ocupa- 
ciones educativas que habrán de conducirle hacia etapas cada vez más plenas y promisoras de mejoramiento. Examinaremos luego el hecho y el ideal educativo en sí mismos, tali como, por una parte se ofrecen y tal como, por otra aparecen como finalidades y aún como normas del ser y del quehacer humanos en sus múltiples facetas y trayectorias. Cotejaremos en seguida las bases y los principios reguladores de la educación. Después habremos de fijarnos especialmente en el tipo de personalidad del eđucador. $\mathrm{Y}$ terminaremos discutiendo la posibilidad y necesidad cle una Filosofía de la Educación Peruana en función del hombre peruano.

De esta manera en el decurso de nuestro filosofar sobre la Educación, subiremos primero a las levantadas cimas đe la teoría, toparemos luego en el suelo de la práctica y nos acercaremos poco a poco al ser humano concreto y vivo, para contemplarlo en relación con aquellos aspectos educativos que apreciamos como más decisivos y preponderantes en los destinos sociales culturales y espirituales del hombre en general y del hombre pertiano en particular.

Así entonces vamos a erigir a la Educación como materia problemática. Ella deberá ser el blanco de nuestra actitud teorética. Si la educación, como dijimos, es un hecho y un ideal, es también y tal vez primero, una dificultad y un problema humano por excelencia, por su contenido, por su intención y por su forma. Por eso es posible una Filosofía de la Educación; y no solamente posible, sino que, sobre todo, necesaria. El futuro del hombre depende de cómo sortee y restuelva la trascendente cuestión y el gran obstáculo que significa el tener urgentemente que educarse. Para ello, se hace indispensable hacer luz en torno, buscar los materiales e instrumentos que sean utilizables y preparar el terreno so- 
bre que debe edificarse la obra de transformación hacia un progreso efectivo y beneficioso. De esto es lo que se ocupa y debe ocuparse la Filosofía de la Educación. Las páginas que vienen hacia ello tienden. Haremos, por tanto, Filosofía, filosofando sobre la educación del ser humano en la tierra.

Lima, Octubre de 1945.

César Góngora Perea.

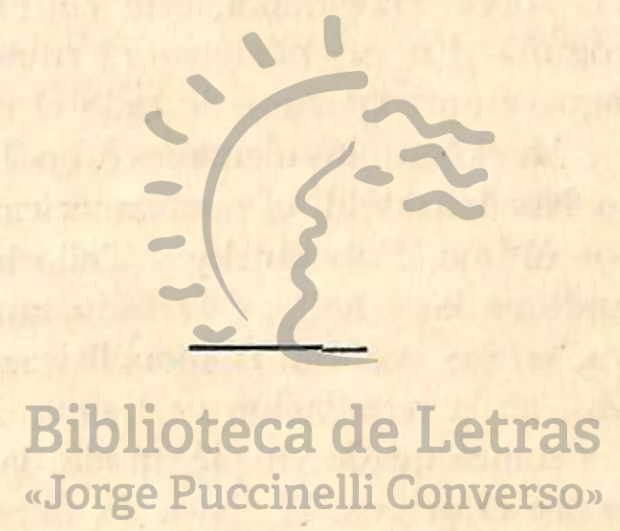

